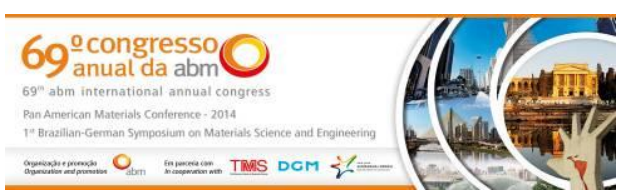

Tema: Gestão de Meio Ambiente e Recuperação e Tratamento de Rejeitos

\title{
AVALIAÇÃO DOS SERVIÇOS AMBIENTAIS PARA RESÍDUOS PERIGOSOS DO SETOR METAL MECÂNICO DO RIO GRANDE DO SUL*
}

\author{
Marise Keller Santos ${ }^{1}$ \\ Ana Curia ${ }^{2}$ \\ Ângela de Moura Ferreira Danilevicz $z^{3}$ \\ Rejane Maria Candiota Tubino 4
}

\section{Resumo}

Este trabalho apresenta os resultados parciais de uma tese de doutorado desenvolvida no PPGE3M da Universidade Federal do Rio Grande do Sul (UFRGS), com as Empresas Produtoras de Resíduos perigosos (EPRs) do setor metal mecânico da região de São Leopoldo/RS e suas Empresas Prestadoras de Serviços Ambientais (PSAs). A maioria das empresas PSAs iniciaram suas atividades com o apoio de grandes EPRs, principalmente, em função das suas necessidades de atender às exigências legais do órgão ambiental estadual, a Fundação Estadual de Proteção Ambiental (FEPAM), para a adequada disposição de resíduos perigosos. É importante salientar que não existe um diagnóstico sobre o setor de prestação de serviços ambientais no RS para resíduos industriais perigosos. Desta maneira, o objetivo deste trabalho foi o de identificar e priorizar as características da qualidade dos serviços ambientais de nove PSAs selecionados pelas próprias EPRs, durante o ano de 2013, por meio da adoção do Quality Function Deployment (QFD). O resultado piloto aponta a proatividade do setor nas características de empreendedorismo e inovação e no atendimento à legislação ambiental.

Palavras-chave: Resíduos perigosos; Setor metal mecânico; Serviços ambientais; QFD.

\section{ASSESSMENT OF ENVIRONMENTAL SERVICES FOR HAZARDOUS WASTE FROM METAL MECHANIC SECTOR OF RIO GRANDE DO SUL}

\section{Abstract}

This paper presents partial results of the doctoral thesis developed in PPGE3M of Universidade Federal do Rio Grande do Sul (UFRGS) with companies producing hazardous waste (EPRs) metal mechanic sector in the region of São Leopoldo/RS and its companies providing environmental services (PSAs). Most PSA's companies started its activities with the support of major EPRs due to their needs to meet the legal requirements of the state environmental agency, Fundação Estadual de Proteção Ambiental (FEPAM) for proper disposal of hazardous waste. It is important to note that does not exist a diagnosis on the sector of environmental services for the RS hazardous industrial waste. The main objective is to identify and prioritize the technical features of the quality of environmental services of nine PSAs selected by their own EPRs, during the year 2013. The methodology used in this assessment is the Quality Function Deployment (QFD) tool applied to assess the quality of services offered by PSAs. The pilot results demonstrate the sector proactivity in the technical characteristics of entrepreneurship and innovation, and environmental compliance.

Keywords: Hazardous waste; Metal mechanical industry; Environmental services; QFD.

1 Engenheira Química, Mestre, Professora, Instituto Federal Sul Rio-grandense, Pelotas, Rio Grande do Sul, Brasil.

2 Engenheira Química, Doutora, Professora, UFRGS, Programa de pós-graduação em engenharia ambiental, Porto Alegre, RS, Brasil.

3 Engenharia civil, Doutora, Professora, PPGEP, UFRGS, Porto Alegre, RS, Brasil; angelamfd@producao.ufrgs.br

4 Engenharia civil, Doutora, Professora, PPGE3M, UFRGS, Porto Alegre, RS, Brasil; rejane.tubino@ufrgs.br

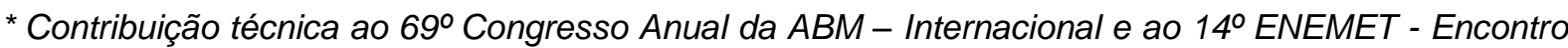
Nacional de Estudantes de Engenharia Metalúrgica, de Materiais e de Minas, 21 a 25 de julho de 2014, São Paulo, SP, Brasil.
} 


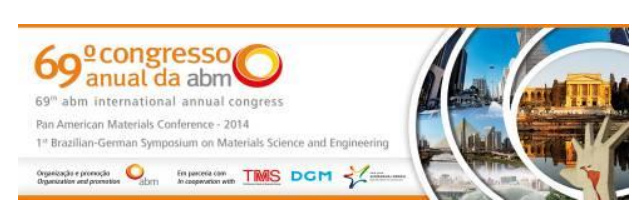

\section{INTRODUÇÃO}

Este trabalho foi desenvolvido como etapa piloto para adequação da metodologia proposta para a avaliação de empresas Prestadoras de Serviços Ambientais (PSAs), tema de doutorado atualmente em desenvolvimento no Programa de PósGraduação em Engenharia de Minas, Materiais e Metalurgia (PPGE3M) da Universidade Federal do Rio Grande do Sul (UFRGS), envolvendo 24 Empresas Produtoras de Resíduos (EPRs) perigosos do setor metal mecânico e eletroeletrônico, do Sindimetal, da região do Vale dos Sinos no Rio Grande do Sul. O trabalho apresenta os resultados da aplicação do método de Desdobramento da Função Qualidade (QFD), do inglês, Quality Function Deployment, para identificação da qualidade dos serviços oferecidos pelas empresas PSAs para resíduos industriais de 24 EPRs. O objetivo principal foi o de identificar e priorizar as características de qualidade dos serviços ambientais de nove empresas PSAs, avaliadas nesta fase piloto e pré-selecionadas pelas próprias EPRs, no ano de 2013. Com isto, esperavase obter as características mais relevantes para gerenciamento, o que permite um futuro planejamento de ações para melhoria da qualidade dos serviços prestados.

O presente artigo desdobra-se em cinco seções, sendo que no primeiro encontramse as diretrizes norteadoras do trabalho. Na segunda seção são apresentados conceitos oriundos do referencial teórico que embasa a realização desta pesquisa, para na seção três serem apresentados os materiais e métodos adotados. Na quarta seção são apresentados os resultados e as discussões acerca dos achados e na quinta e última seção são apresentadas as considerações finais e sugestões de trabalhos futuros.

\section{SERVIÇOS AMBIENTAIS E O QFD}

A definição empregada pela Organisation for Economic Cooperation and Development (OECD) no documento da Eurostat [1] para serviços ambientais especificados no manual da indústria ambiental, identifica serviços ambientais como aqueles que:

envolvem atividades de medir, prevenir, limitar, minimizar ou corrigir danos ambientais a água, solo, bem como problemas relacionados a resíduos, ruídos e eco sistemas. Isto inclui tecnologias limpas, bens e serviços que reduzam os riscos ambientais e minimizem a poluição e uso de recursos.

Em complemento, define o setor de bens e serviços ambientais como:

um conjunto heterogêneo de tecnologias, bens e serviços que: medem, controlam, restauram, previnem, tratam, minimizam, conscientizam e pesquisam danos ambientais ao ar, água, solo bem como aos problemas relacionados a ruídos, resíduos, biodiversidade e à paisagem.

O setor de serviços ambientais no Brasil para resíduos perigosos não está diagnosticado na sua totalidade por parte das autoridades do setor público e privado. Entretanto, existem iniciativas no intuito de analisar este mercado, como o trabalho realizado no Estado do Espírito Santo, através do Relatório final da pesquisa de Oferta de Bens e Serviços Ambientais [2], o documento Diagnóstico Brasileiro de Emprego Verde da Organização Internacional do Trabalho [3] e o Perfil do Setor de Tratamento de Resíduos e Serviços Ambientais, elaborado pela Associação Brasileira de Empresas de Tratamento de Resíduos - ABETRE [4]. Mais especificamente no Estado do Rio Grande do Sul, uma das maiores barreiras encontradas para o desenvolvimento do setor de serviços ambientais encontra-se na

\footnotetext{
* Contribuição técnica ao 69ำ Congresso Anual da ABM - Internacional e ao 14ํㅡㄹ ENEMET - Encontro Nacional de Estudantes de Engenharia Metalúrgica, de Materiais e de Minas, 21 a 25 de julho de 2014, São Paulo, SP, Brasil.
} 


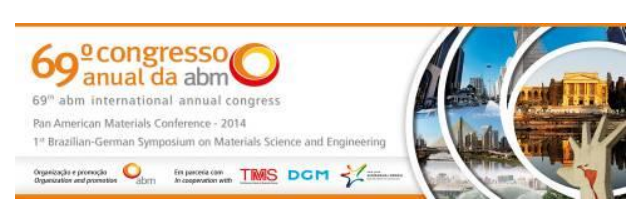

falta de informações sobre as quantidades dos resíduos produzidos pelas EPRs. A última informação divulgada pela Fundação Estadual de Proteção Ambiental Henrique Roessler (FEPAM) foi no ano de 2002, no documento intitulado Inventário Nacional de Resíduos Sólidos, contendo dados sobre resíduos, efluentes e emissões produzidos pelos setores industriais [5]. Desta maneira, estas informações estão desatualizadas, considerando, principalmente, as mudanças no cenário econômico no Estado em função da alteração do número de empresas e do consequente volume e das características dos resíduos produzidos.

Considerando uma empresa (PSA), como um dos fornecedores de serviços para os resíduos de qualquer empresa que produza resíduos e que estes devam ser avaliados na gestão da cadeia de abastecimento verde de uma empresa certificada, pode-se afirmar que os PSAs deverão cumprir com todos os requisitos de qualidade, ambientais e de segurança e saúde ocupacional estabelecidos em um sistema de avaliação de fornecedores, conforme seus integrantes. Na Figura 1 é possível identificar os fluxos tradicionais de resíduos para a sua adequação ambientalmente correta, tradicionalmente empregada pelas EPRs, considerando o enfoque previsto na Política Nacional de Resíduos Sólidos (PNRS) [6].

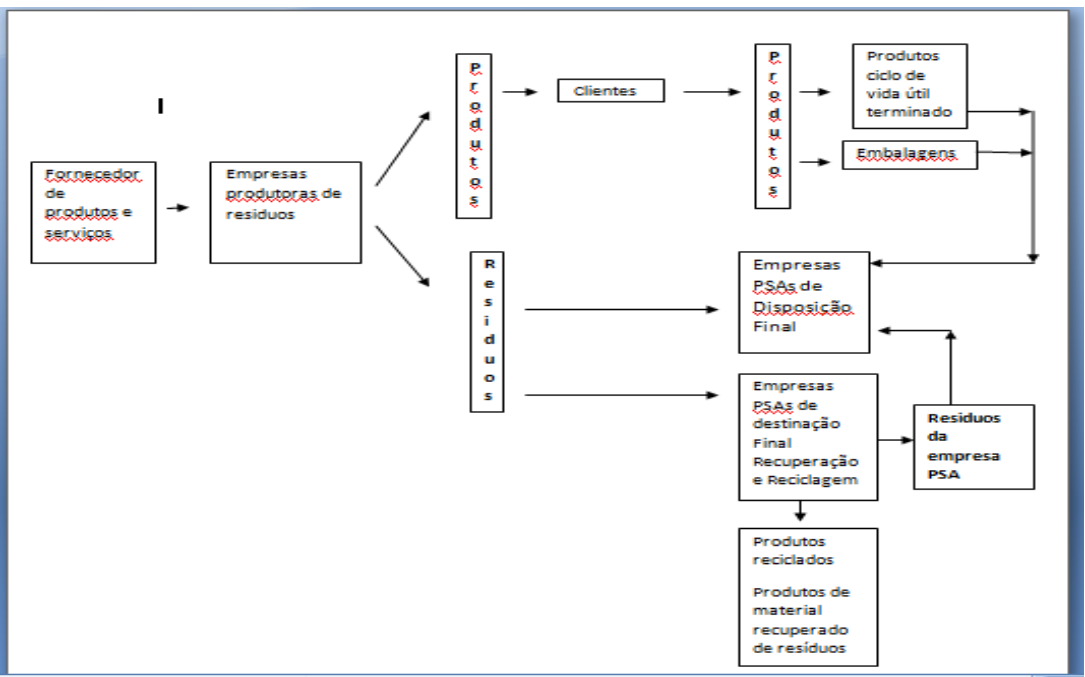

Figura 1: Principais Integrantes da cadeia de fornecedores de uma empresa produtora de resíduos e produtos.

As exigências das EPRs sofrem uma grande influência decorrente da responsabilidade compartilhada, definida pela Política Nacional de Resíduos Sólidos (PNRS) [6], a qual estabelece as relações entre EPRs e PSAs. A consequência imediatamente observada é a necessidade do setor de serviços ambientais aprimorar, constantemente, a qualidade técnica de seus serviços, mas também implementar uma adequada gestão ambiental para os resíduos, emissões e efluentes produzidos na execução de seus serviços.

Em muitos aspectos a empresa prestadora de serviços assemelha-se a uma indústria tradicional: consome recursos, água e energia e gera efluentes líquidos, resíduos sólidos e emissões atmosféricas, mas a grande diferença é que estes impactos são causados não pela fabricação de produtos, mas sim pela prestação de serviços para resíduos. Dessa forma, o grande desafio para o setor ambiental é minimizar o emprego de recursos como água e energia e a geração de resíduos, melhorando continuamente a qualidade dos serviços oferecidos, com o menor impacto ambiental.

\footnotetext{
* Contribuição técnica ao 69ำ Congresso Anual da ABM - Internacional e ao 14ํㅡㄹ ENEMET - Encontro Nacional de Estudantes de Engenharia Metalúrgica, de Materiais e de Minas, 21 a 25 de julho de 2014, São Paulo, SP, Brasil.
} 
Segundo a Cepal [7], em processo de observação às empresas PSAs na América Latina, caracterizadas enquanto prestadores de serviços de reciclagem e/ou recuperação de resíduos, e, considerando que os resíduos são sua matéria prima, identificou a necessidade de definição de um padrão mínimo de qualidade de entrega dos resíduos gerados pelas EPRs, de maneira que os mesmos pudessem ser recuperados ou reciclados pelas tecnologias empregadas pelas empresas PSAs. A definição estratégica de avaliação de fornecedores, incluindo as empresas PSAs, tem o objetivo de desenvolver esses fornecedores para que tenham a capacidade e a intenção de serem empresas parceiras em longo prazo e na busca da excelência na oferta de seus serviços.

Segundo Duarte [8], as empresas de grande porte certificadas pela norma NBR ISO 9001:2008 [9] qualificam seus fornecedores e esse credenciamento significa o reconhecimento do atendimento aos requisitos mínimos necessários para o estabelecimento de relações comerciais. Esse reconhecimento se dá com base na capacidade de um fornecedor fornecer materiais e serviços em conformidade com as especificações técnicas exigidas por seus clientes, além de atender aos requisitos legais vigentes e específicos de contratos estabelecidos.

A seleção de um fornecedor parte do princípio que este possui capacidade de atender aos requisitos contratuais, estabelecido segundo preço competitivo, prazo de entrega e requisitos de qualidade dos materiais ou serviços a serem entregues.

Além disto, muitas vezes, as empresas com certificação em sistemas de gestão auditam seus fornecedores, através de seus próprios colaboradores (auditores internos) capacitados para este objetivo que são, então, denominados de auditores de segunda parte.

A aplicação do QFD na priorização das características de qualidade dos serviços a serem avaliados, considera o modelo desenvolvido para a utilização em serviços, composto pela matriz da qualidade [10]. A construção da matriz da qualidade é elaborada a partir dos resultados da pesquisa de mercado das demandas da qualidade definidas pela voz do cliente. Neste modelo o cliente é definido como as EPRs. Pelo QFD é possível identificar, através das demandas do cliente, neste caso as EPRs, as necessidades e desejos desse público quanto a qualidade dos serviços ambientais. É possível ainda refletir sobre o que uma EPR espera de um serviço ambiental e o que mais valoriza na oferta deste serviço. Ainda, segundo Osório [11] a aplicação da ferramenta QFD para avaliação de fornecedores, pode ser empregada para qualquer tipo de organização, de serviços ou de manufatura de produtos, sempre que os problemas apresentem muitos critérios distintos para sua avaliação.

A seleção de fornecedores em uma empresa pode ser tomada como uma decisão multicritério, sendo necessário o emprego de ferramentas como o QFD, para uma melhor avaliação de todos os fatores que influenciam na decisão, considerando também as preferências do "cliente". A avaliação de desempenho de fornecedores deve ser um processo flexível e que permita a melhoria contínua do planejamento elaborado a partir dos resultados das avaliações realizadas. $O$ objetivo do resultado destas avaliações é permitir futuras negociações com os fornecedores.

Segundo Ferreira e Ribeiro [10] o setor de Serviços caracteriza-se por uma forte componente subjetiva, significativamente importante para sua avaliação, este fato dificulta o emprego de critérios subjetivos que possam ser facilmente quantificáveis. O método QFD, empregando a análise da importância corrigida da qualidade demandada, das características de qualidade, e sua priorização, obtém resultados considerando também o emprego de características subjetivas, que facilitam o

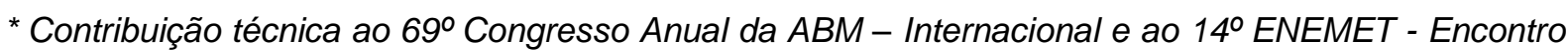
Nacional de Estudantes de Engenharia Metalúrgica, de Materiais e de Minas, 21 a 25 de julho de 2014, São Paulo, SP, Brasil. 
processo de tomada de decisões inerente ao Planejamento da Qualidade de serviços.

\section{MATERIAIS E MÉTODOS}

O público alvo, clientes, no caso deste trabalho, são 24 EPRs do setor metal mecânico e eletro eletrônico, integrantes do Comitê de Meio Ambiente e Saúde e Segurança (SSMA) do Sindimetal da cidade de São Leopoldo no Rio Grande do Sul. Os profissionais representantes da área ambiental e de saúde e segurança destas empresas, denominados de avaliadores, foram preparados para a aplicação desta metodologia para a avaliação de empresas PSAs.

Os 24 EPRs identificaram suas empresas PSAs para a destinação final de seus resíduos perigosos, classe I, segundo a NBR 10004 (ABNT, 2004) [12]. Inicialmente optou-se pela realização de um piloto, realizado com 09 empresas PSAs, comuns às 24 EPRs. A Tabela 1 caracteriza as empresas PSAs.

Tabela 1: Caracterização das empresas PSAs que integraram a fase piloto

\begin{tabular}{l|l|c}
\hline \multicolumn{1}{c|}{ Empresa PSAs } & \multicolumn{1}{c}{ Serviço ambiental } & $\begin{array}{c}\text { Numero de } \\
\text { empresas } \\
\text { PSAs }\end{array}$ \\
\hline Central de resíduos & Disposição final de resíduos & 3 \\
\hline Transporte de resíduos & Transportador de resíduos & 1 \\
\hline Recuperação de resíduos & Higienização de uniformes e EPls & 1 \\
& Higienização de embalagens & 1 \\
\hline Reciclagem & Reciclagem de material eletroeletrônico & 1 \\
& Reciclagem de óleos usados & 1 \\
& Reciclagem de resíduos líquidos & 1 \\
\hline
\end{tabular}

Considerando a aplicação da ferramenta QFD e a construção da matriz da qualidade, para o desenvolvimento da metodologia, as etapas para a execução do trabalho foram baseadas nas informações apresentadas no livro de Ribeiro, Echeveste e Danilevicz [13], as quais são apresentadas a seguir:

- Desdobramento da qualidade demandada

- Priorização da qualidade demandada (IDi*)

- Desdobramento das características da qualidade demandada

- Relacionamento da qualidade demandada com as características de qualidade

(DQij)

- Especificações atuais para as características de qualidade

- Importância das características de qualidade (IQj)

- Avaliação da dificuldade de atuação sobre as características de qualidade (Dj)

- Avaliação competitiva das características de qualidade (Bj) e (Mi)

- Priorização das características de qualidade (IQj $)$

$\mathrm{Na}$ aplicação do QFD, inicialmente, foi realizada uma pesquisa de mercado, com o objetivo de identificar as demandas de qualidade junto aos clientes do serviço, as EPRs. O objetivo era o de dar a destinação ambientalmente adequada para seus resíduos industriais e seu transporte, avaliando o cumprimento da legislação ambiental, de saúde e segurança vigentes e aplicáveis aos procedimentos da gestão de serviços oferecidos pela empresa PSA.

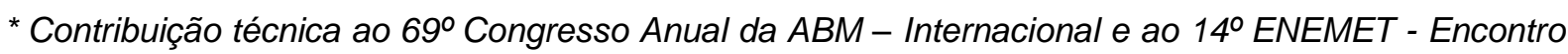
Nacional de Estudantes de Engenharia Metalúrgica, de Materiais e de Minas, 21 a 25 de julho de 2014, São Paulo, SP, Brasil.
} 


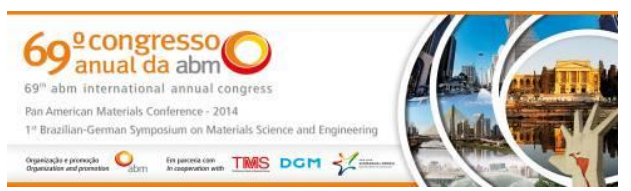

O período para a realização da avaliação das 09 empresas PSAs piloto, foi definido em três meses, período no qual, foram realizados para cada PSA avaliado, três encontros com os auditores responsáveis e as autoras deste trabalho. O primeiro encontro teve o objetivo de preparar a avaliação com a documentação solicitada previamente e enviada pela empresa PSA. O segundo encontro realizado nas instalações do PSA, objetivou a avaliação da documentação complementar e as próprias instalações e os procedimentos na oferta dos serviços ambientais pelo PSA. O último encontro avaliou as informações obtidas e estabeleceu um consenso em relação aos quesitos entre os avaliadores e as autoras deste trabalho. Também teve enquanto mote colher sugestões dos envolvidos no processo de aperfeiçoamento da metodologia proposta.

As principais características de cada etapa para a construção da matriz da qualidade são descritas na sequência deste artigo.

\subsection{Desdobramento da Qualidade Demandada}

Identifica o cliente, no presente trabalho, representado pelas 24 EPRs integrantes do Comitê de SSMA e estabelece as demandas da qualidade para serviços ambientais de resíduos industriais perigosos.

As demandas de qualidade foram identificadas junto à 24 EPRs, através de seus representantes técnicos pelas áreas de SSMA, e definida a importância de cada uma das demandas. A seguir são apresentadas as demandas em sua ordem de importância.

a) Legais: aplicáveis aos processos de licenciamento e de gestão de atividades da prestação de serviços ambientais.

b) Riscos: avaliam os riscos das atividades características na execução dos serviços ambientais oferecidos pelos PSAs nos resíduos industriais de EPRs.

c) Qualidade: são relacionados à qualidade da gestão operacional dos serviços ambientais e transportes dos resíduos oferecidos às EPRs relacionados às atividades de monitoramento na recepção dos resíduos e do fluxo dos serviços aplicados aos resíduos.

d) Saúde e segurança ocupacional: são ações que caracterizam a gestão de um sistema de saúde e segurança aplicados no ambiente de trabalho e na realização de atividades dos colaboradores que atuam na empresa PSA e suas instalações.

e) Gerenciamento de águas, efluentes, ruídos e emissões do PSA: são relacionados ao uso da água pelo PSA, geração e tratamento de efluentes líquidos, ruídos e emissões atmosféricas geradas, na execução do serviço ambiental no resíduo industrial do EPR.

f) Gerenciamento de resíduos do PSA: são relacionados às ações relacionadas às alternativas para a disposição e destinação final, considerando os critérios da PNRS, dos resíduos gerados pelo próprio PSA na execução do serviço ambiental no resíduo industrial do EPR.

g) Critérios de tecnologia: avaliação relacionada à tecnologia empregada pelo PSA para os resíduos do EPR, considerando as prioridades de ações estabelecidas na PNRS para a destinação ambientalmente adequada para resíduos.

h) Gestão empresarial: são relacionadas às características da gestão empresarial vinculadas a ações que são refletidas no marketing empresarial e financeira.

\footnotetext{
* Contribuição técnica ao $69^{\circ}$ Congresso Anual da ABM - Internacional e ao 14ํㅡㄹ ENEMET - Encontro Nacional de Estudantes de Engenharia Metalúrgica, de Materiais e de Minas, 21 a 25 de julho de 2014, São Paulo, SP, Brasil.
} 


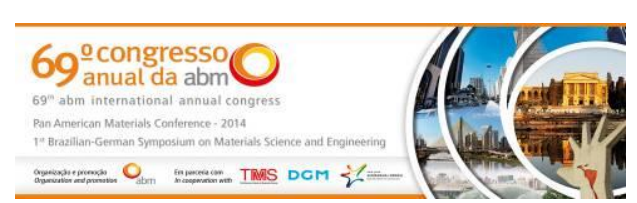

i) Gestão da inovação e empreendedorismo: são relacionadas às características inovadoras do PSA na busca do aperfeiçoamento dos atuais serviços ambientais oferecidos e na oferta de novos serviços ambientais para resíduos industriais, que possam atender as prioridades estabelecidas na gestão de resíduos na PNRS.

\subsection{Priorização da Qualidade Demandada IDi*}

A priorização é calculada considerando a importância de cada item da qualidade demandada (IDi) atribuído pelas EPRs a cada demanda, considerando a correção da priorização utilizando-se o valor de uma avaliação estratégica (Ei) e competitiva (Mi) da empresa PSA.

Os valores de Ei para cada demanda da qualidade são obtidos considerando a seguinte escala [13]:

- 0,5 Importância pequena

- 1,0 Importância média

- 1,5 Importância grande

- 2,0 Importância muito grande

A atribuição destes índices a cada demanda da qualidade considerando a avaliação estratégica (Ei) do PSA em relação a outras PSAs do mesmo segmento significa o impacto do atendimento da demanda especificada na preservação da imagem, competitividade e manutenção no mercado de serviços ambientais do PSA.

Os valores da avaliação competitiva (Mi) de cada qualidade demandada são obtidos utilizando-se a seguinte escala [13]:

- 0,5 Acima da concorrência

- 1,0 Similar a concorrência

- 1,5 Abaixo da concorrência

- 2,0 Muito abaixo da concorrência

Esta avaliação considera uma comparação entre o atendimento do PSA às demandas de qualidade do EPR, e como os outros PSAs concorrentes no mesmo segmento de serviços ambientais conseguem ou não atender a esta qualidade demandada.

A equação (1) utilizada para realizar a correção do índice de importância da qualidade demandada empregando os valores de Ei e Mi é [13]:

Sendo:

$$
\mathrm{IDi}^{*}=\mathrm{IDi} \times \sqrt{\mathrm{Ei}} \times \sqrt{\mathrm{Mi}}
$$

$\mathrm{IDi}^{*}=$ Índice de importância corrigido da qualidade demandada

IDi = Índice de importância da qualidade demandada

$\mathrm{Ei}=$ Avaliação estratégica de cada qualidade demandada pelas EPRs

$\mathrm{Mi}=$ Avaliação competitiva dos itens da qualidade demandada (benchmark)

\subsection{Desdobramento das Características da Qualidade (Indicadores de Qualidade)}

Para cada qualidade demandada foram identificados um ou mais indicadores que compõem o conjunto de características de qualidade. Estas características são utilizadas para traduzir as demandas em requisitos técnicos, mensuráveis e objetivos. As mesmas são avaliadas nas empresas PSAs com o objetivo de

\footnotetext{
* Contribuição técnica ao $69^{\circ}$ Congresso Anual da ABM - Internacional e ao 14ํㅡㄹ ENEMET - Encontro Nacional de Estudantes de Engenharia Metalúrgica, de Materiais e de Minas, 21 a 25 de julho de 2014, São Paulo, SP, Brasil.
} 


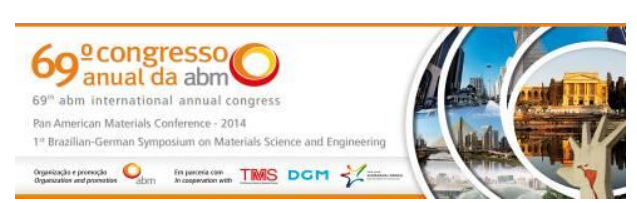

Sendo:

IQj = Importância das características de qualidade considerando os aspectos técnicos dos serviços ambientais

$\mathrm{IDi}^{*}=$ Índice de importância corrigido da qualidade demandada dos serviços ambientais

DQij = Intensidade do relacionamento entre os itens da qualidade demandada e das características (indicadores) dos serviços ambientais.

\subsection{Avaliação da Dificuldade de Atuação sobre as Características de Qualidade Dj}

Nesta etapa considera-se a dificuldade de modificar um indicador associado a uma demanda de qualidade especificada para um serviço ambiental pela EPR. A avaliação utiliza a escala [13]:

- 0,5 muito difícil

- 1,0 difícil

- 1,5 moderado

- 2,0 fácil alterar

\subsection{Avaliação Competitiva das Características da Qualidade Bj e Mi}

Nesta etapa é considerada a influência da concorrência, outras empresas PSAs do mesmo segmento, consistindo no denominado Benchmark técnico.

A escala a ser atribuída ao índice de Bj é [13]:

- 0,5 acima da concorrência

- 1,0 Similar à concorrência

- 1,5 Abaixo da concorrência

- 2,0 Muito abaixo da concorrência

O benchmark comercial é considerado na avaliação do índice Mi com a seguinte escala [13]:

- 0,5 Acima da concorrência

- 1,0 Similar a concorrência

- 1,5 Abaixo da concorrência

- 2,0 Muito abaixo da concorrência

O objetivo é avaliar o quanto as demandas da qualidade estão defasadas em relação à concorrência sempre comparando as empresas PSAs no mesmo segmento de atuação.

\subsection{Priorização das Características da Qualidade IQj*}

Para a priorização das características da qualidade utiliza-se nesta etapa a seguinte equação 3 [13]:

Sendo:

$$
I Q j^{*}=I Q j \times \sqrt{ } \mathrm{Dj} \times \sqrt{ } \mathrm{Bj}
$$

IQj ${ }^{\star}=$ importância corrigida das características da qualidade

$\mathrm{IQj}$ = Importância das características da qualidade considerando a importância técnica do indicador para serviços ambientais

$\mathrm{Dj}=$ Avaliação da dificuldade de atuação

$\mathrm{Bj}=$ Avaliação da competitividade considerando os aspectos técnicos do indicador em relação aos concorrentes.

* Contribuição técnica ao $69^{\circ}$ Congresso Anual da ABM - Internacional e ao 14ํㅡㄹ ENEMET - Encontro Nacional de Estudantes de Engenharia Metalúrgica, de Materiais e de Minas, 21 a 25 de julho de 2014, São Paulo, SP, Brasil. 


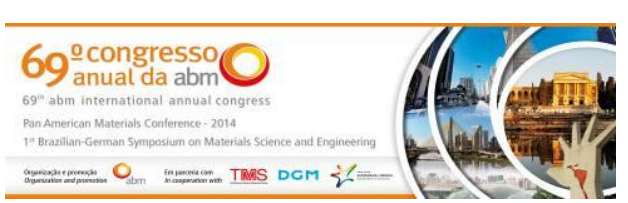

\section{Agradecimentos}

As autoras agradecem aos profissionais da empresa Stihl e Hygra e também ao Sindimetal pela oportunidade de trabalhar em conjunto com suas empresas associadas. Um agradecimento especial ao CNPq pela bolsa de doutorado recebida.

\section{REFERÊNCIAS}

1 Eurostat, European Comission. The Environmental Goods and Services. Methodologies and Working papers. Manual 25. 2009.

2 Teixeira TCMR, Santos CV. Relatório Final - Pesquisa de Bens e Serviços Ambientais do Estado do Espírito Santo. Vitória; 2010.

3 Moçouçah PS. Empregos Verdes no Brasil. Quantos são, onde estão, e como evoluirão os próximos anos. Brasil: Organização Internacional do Trabalho; 2009.

4 Associação Brasileira de Empresas de Tratamentos de Resíduos. Perfil do Setor de Tratamento de Resíduos e Serviços Ambientais. ABETRE; 2006.

5 FEPAM. Inventário Nacional de Resíduos Sólidos, Etapa Rio Grande do Sul. Fundação estadual de Proteção Ambiental Henrique Roessler. FEPAM apoio Ministério do Meio Ambiente; 2002.

$6 \quad$ PNRS. Brasil. Lei Federal n. 12.305 de 02 de agosto de 2010. Institui a Política Nacional de Resíduos Sólidos; altera a Lei no 9.605, de 12 de fevereiro de 1998; e dá outras providências. Brasília, 02 de agosto de 2010.

7 CEPAL. Comisión Económica para América Latina y el Caribe (CEPAL) y Deutsche Gesellschaft für Technische Zusammenarbeit (GTZ). Las pymes en el mercado de bienes y servicios ambientales: identificación de oportunidades, políticas e instrumentos Estudios de caso de: Argentina, Chile, Colombia y México. Naciones Unidas. 2006.

8 Duarte. IG. A Norma ISO 14001 e a Relação com os Fornecedores [dissertação de mestrado]. Departamento de Economia, Gestão e Engenharia Industrial, Universidade de Aveiro; 2009.

9 Associação Brasileira de Normas Técnicas. NBR/ISO 9001: Sistemas de gestão da qualidade- Requisitos. Rio de Janeiro; 2008.

10 Ferreira AM, Ribeiro JLD. O uso do QFD no gerenciamento de projetos: um estudo de caso no setor de serviços. In: Anais do 15 Encontro Nacional de Engenharia de Produção; 1995; São Carlos, Brasil. São Carlos: UFSCar; 1995. v.1, p.507-512.

11 Osório CJ, Arango DC, Ruales CE. Selección de proveedores usando el despliegue de la función de calidad difusa. Revista EIA. 2011;Julio(15):73-78.

12 Associação Brasileira De Normas Técnicas. NBR 10.004: Resíduos Sólidos Classificação. Rio de Janeiro; 2004.

13 Ribeiro JLD, Echeveste MES, Danilevicz AMF. A utilização do QFD na otimização de produtos, processos e serviços. Porto Alegre: Feeng, UFRGS; 2001.

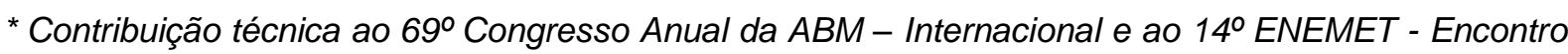
Nacional de Estudantes de Engenharia Metalúrgica, de Materiais e de Minas, 21 a 25 de julho de 2014, São Paulo, SP, Brasil.
} 